\title{
XIV.
}

Aus der Kinderklinik, dem pathologischen, pharmakologisehen und physiologischen Institut zu Heidelberg.

\section{Über einen Fall von akuter Degeneration des Leberparenchyms.} Von

\section{Franz Soetbeer.}

Unter Mitarbeit von Otto Cohnheim, Edgar Gierke, Martin Jacoby, Jussuf Ibrahim und Hermann Steudel.

Im November 1901 wurde ein schwer komatöser Patient in die Kinderklinik eingewiesen. Anamnese und Symptome wiesen auf akute Erkrankung der Leber hin. Die Untersuehung des Harns auf Ammoniak nach Nencki ${ }^{1}$ ) ergab nach 5 Stunden brauchbare Ammoniakwerte, die klar für die Vermebrung des $\mathrm{NH}_{3}$ im Vergleich zum Gesamtstickstoff sprachen; man konnte also annehmen, daß eine intensivere Säuerung des Organismus wahrscheinlich war und riehtete den Versuchsplan mit Rücksicht auf die gefundene Tatsache ein.

Es war ganz gleichgtiltig, welche klinische oder anatomisehe Diagnose später gestellt wurde, es mufte sofort die umfassende Beobachtung einsetzen, um nicht später Lücken des Materials bedauern zu müssen.

Um die rasche Beobachtung und die Sieherheit der Resultate, die mit der Erfabrung des Beobachters wäehst, nicht zu gefährden, taten wir uns zusammen, und jeder von uns untersuchte das ihm methodiseh nach seiner früheren Arbeitsrichtnng am besten liegende Gebiet.

Die Krankheit endete letal, und wir geben in folgendem das Erschaute wieder. Als Grundlage für unsere Darstellung haben wir den anatomischen Bericht anzusehen, denn dieser allein gibt uns mit Sicherbeit die Lokalisation, die Intensität und den Umfang der Erkrankung an.

1) Nencki und Zaleski, dieses Archiv. Bd. XXXVI. S. 395. 
Die Natur unseres Falles ist leider dunkel geblieben, und auch sonst sind die Ergebnisse unserer Untersuchungen nicht derart ausgefallen, daß sie eine Erweiterung unseres Wissens bringen. Doch glauben wir, daß der Untersuchungsplan für ähnliche klinische $V_{\text {or- }}$ kommnisse vorbildlich sein kann, und deshalb tibergeben wir ihn der Öffentlichkeit.

Anatomische Untersuchung (Gierke).

Dem Sektionsprotokolie (Dr. Fischler) entnehme ich folgenden Auszug: ziemlich großes Kind mit starkem Hydrops (Gesicht, Rücken, Skrotım, Beine, Arme), ziemlich starker Ikterus der Haut und der sichtbaren Schleimbäute.

In der freien Peritonealhöhle ca. $1200 \mathrm{ccm}$ eines mit Fibrinflocken untermischten, leicht gelben Ergusses. Starke Blähung des Magens und des Kolon; die Leber ist unter dem Rippenbogen versehwunden. Im kleinen Becken sind die etwas injizierten Dämme geringgradig durch Fibrinflocken verklebt; das Netz ist sulzig, Mesenterialdrüsen nicht vergrößert.

Blut bräunlichrot, langsam gerinnend. Unter dem Epikard einige kleine Hämorrhagien. Herzmuskelfeisch blaß, gut kontrahiert; an Herzklappen und Endokard kein auffälliger Befund.

Beiderseits, besonders links, Pleuraadhäsionen und subpleurale Hämorrhagien. In beiden Unterlappen zum Teil konfluierende broncho. pneumonische Herde.

Milz etwas vergrößert; auf dem Durchschnitt mit deutlicher Follikelzeichnung und braunroter Pulpa.

Die Leber ist stark verkleinert, von grüngelber Farbe, mit höckerigen Veränderungen auf ihrer Oberfäche, die von Linsen- bis Haselnußgröße schwanken und den linken Lappen bedeutend mehr betreffen als den rechten. Der Lobus caudatus ist relativ groß gegen die andern und von grasgrüner Farbe. Die Konsistenz der Leber ist derb, es fällt Vermehrung des Bindegewebes schon makroskopisch auf, namentlich auf dem Durchschnitt. Gerade hier zeigt sich eine sehr verschiedene Verteilung der derben Bindegewebswucherung auf die einzelnen Lappen und da wieder auf die nicht als Knoten und Höcker imponierenden Teile. Der Lobus caudatus ist offenbar am wenigsten verändert, nicht derb, mit zentroacinärer, weiblicher Zeichnung und hochgradigem Ikterus, der sich überhaupt scharf an die Leberparenchymreste, welche durch die Knoten und Höcker repräsentiert werden, auch in der übrigen Leber hält. Die Porta hepatis bietet keine Veränderungen dar. Die Gallenblase ist ziemlich stark gefüllt, etwas dickwandig und enthält zähflüssige, helle Galle. Die großen.Gallenwege bieten keine Besonderheiten.

Die Nieren etwas vergrößert, die Kapsel leicht abziehbar; Nierenoberfläche glatt und spiegelnd. Auf dem Durchschnitt Mark und Rinde regelmäßig abgegrenzt, die Papillenspitzen von gelber; fettiger Farbe; die Rinde etwas getrübt.

Gehirnsubstanz sehr ödematös. Windungen völlig glattgedrückt, Furchen fast ganz verstrichen. 
Nach dem makroskopischen Befunde wurde folgende anatomische Diagnose gestellt: Hochgradige Cirrhosis hepatis (toxica?), Bronchopneumonien in beiden Unterlappen. Pleuritis adhaesiva duplex. Hämorrhagien in Perikard und Pleuren. Gehirnödem. Hydrops anasarea und Aszites. Trübung der Nierenrinde. Geringer Milztumor. Ikterus.

Die derbenPartien der Leber, die sich nach Formolhärtung noch schärfer in weißlicher Farbe gegen die dunkelgruin gewordenen ikterischen Stellen abgrenzen, sind mit einer ganz enormen Bindegewebswucherung umgeben. Wir haben große Partien vor uns, in denen anf den ersten Blick nichts von Lebergewebe zu vermuten ist. Ovale bis spindelförmige große Zellen mit spärlicher Interzellularsubstanz, durchzogen von einem weitmaschigen Kapillarnetze, hier und da leakozytäre Elemente (meist Lymphozyten, seltener polynukleäre und eosinophile Zellen) enthaltend, werden durch schlauchförmig sich durchflechtende Epithelstränge, zum Teil mit deutlichem Lumen, in unregelmäßige, rundliche oder polygonale Gruppen geteilt. Hier und da gelingt es, in diesen Bindegewebsmassen eine ikterische, mit Sudanfärbung isoliert sich stark rötende, typische Leberzelle nachzuweisen. Man kann das hier sich bietende Bild kurz folgendermaßen definieren: Die Acini sind völlig durch Massen jungen Bindegewebes ersetzt, das zwischen sich noch ganz vereinzelte degenerierte Leberzellen einschließt. Die acinöse Struktur kommt dadurch deutlich zum Ausdruck, daß die ganze Peripherie des Acinus von einer Zone starker Gallengangswucherung eingenommen wird. (Über die Schwierigkeit der Unterscheidung zwischen neugebildeten Gallengängen und Leberzellresten vgl. Barbacci, Über Ausgang der aknten Leberatrophie in multiple, knotige Hyperplasie, Zieglers Beiträge, Bd. XXX. Heft 1.) Auf Grund dieser Auffassung werden wir Gefäße, die zwischen diesen gewucherten Gallengängen anzutreffen sind, als dem portalen Systeme zugehörig betrachten, während die in der Mitte der Bindegewebshaufen sich sammelnden Gefäße den Lebervenen entsprechen.

Wenden wir uns nun von diesem einen Extreme, der fast völligen bindegewebigen Umwandlung von Lebersubstanz, dem anderen zu, das sich uns in den weichen ikterischen Knoten bietet: deutliche Acinusstruktur durch die Verschiedenheit der zentroacinären und der peripherischen Zellen scharf markiert, ohne jede Bindegewebsvermehrung, bedingt hier den Aufbau. Unverkennbare Leberzellen setzen den ganzen Acinus zusammen, in den zentralen Zonen ikterisch nur mit Fetttropfen mannigfachster Größe überladen, in den peripheren Zonen trübe geschwollen und nur mit einzelnen Fetttropfen besetzt. Zeichen von lebhafterer Neubildung (Mitosen), wie man sie in Adenomknoten findet, sind nicht vorhanden. Zwischen den Leberzellen fallen häufig erweiterte, mit dunkelgrünem Inhalt gefüllte Gallenkapillaren auf. Die Vena centralis ist meist deutlich ausgeprägt, weder um sie, noch um die Pfortaderäste ist Bindegewebswucherung nachweisbar.

Zwischen diesen so völlig verschiedenen Partien ist es nun nicht schwierig, Übergänge zu finden, da die Grenze der parenchymatösen, knotenförmigen gegen die bindegewebigen Stellen keine ganz scharfe ist. Dort sehen wir dann in verschiedener Abstufung interacinäre Gallengangs- 
wucherung und intraacinäre Bindegewebsnenbildung mit Schwund und Degeneration der spezifischen Leberzellen einhergehen.

Die Nieren, die auch schon makroskopisch Trübung der Rinde aufwiesen, lassen mikroskopische Trübung und Schwellung der Epithelien, wenn auch im bescheidenen Maße, nicht vermissen. Dazu finden sich die Epithelien sowohl beider Arten von gewundenen wie der geraden Harnkanälchen ziemlich allgemein mit deutlich granulärem Fett erfüllt. In dem einen oder anderen Harnkanälchen findet sich auch einmal ein hyaliner oder leicht gekörnter Zylinder. Stärkere desquamative oder interstitielle Prozesse fehlen.

Die Milz bietet auch mikroskopisch follikuläre Hyperplasie; in dem in fast jedem Malpighischen Körperchen deutlich ausgeprägten Keim* zentrum sind Mitosen spärlich vorhanden, dagegen fallen auf zwischen den lichten Zellen intensiv mit Hämatoxylin gefärbte, unregelmäßige, kleine Gebilde, die wohl nur als Kernfragmente gedeutet werden können.

In den Lungen finden sich leukozytäre Broncho-Pnenmonien mit Hyperämie, keine Bindegewebsvermehrung.

Wenn wir die Ergebnisse der mikroskopisehen Untersuchung kurz zusammenfassen, so finden wir in der Leber einen Prozel, der einerseits Knoten von typisch acinärem Bau mit starker Trübung; Ikterus und intensiver Fettdegeneration der Leberzellen erzeugt hat, andererseits dazwischen Zonen exzessiver Bindegewebsvermebrung, die zuletzt zu völligem Ersatze des Leberacinus durch Bindegewebe und zu periaoinärer Gallengangswucherung geführt hat, hervortreten läBt. Dazu gesellen sich parenchymatöse Prozesse in den Nieren, geringe Veränderungen der Milz und leukozytäre Bronchopneumonien.

Betreffs der Ätiologie ist nicht viel Sicheres auszusagen. Die Erkrankung, die uns bei ausgedehnten interstitiellen Prozessen zuerst in den Sinn kommt - ich meine die Syphilis, bei der ja auch eine parenchymatöse Degeneration durch Toxinwirkung nicht ausgeschlossen erscheint - , erfährt hier jedenfalls weder durch die Krankengeschichte noch durch die sonstigen anatomischen Befunde (die Knochen konnten leider aus äußeren Gründen nicht untersucht werden) eine Stätze.

Andere primär-interstitielle Prozesse kommen noch weniger in Betracht; die versehiedenen Formen der Leberzirrhose bieten nur oberflächliche Ähnlichkeiten, für biliäre Zirrhose, an die der Ikterus und die Gallengangswucherung am ehesten noch denken lassen, liegt gar kein Anhaltspunkt vor, da die Präparation der Gallengänge ihre völlige Wegsamkeit ergab.

Von plimär-parenchymatösen Prozessen erinnert in vielem der Ausgang der akuten gelben Leberatrophie, den Ziegler in seinem Lehrbuche beschreibt, an unsern Fall; nur scheint bei letzterem die Verteilung eine unregelmäBigere zu sein. Eine knotige Hyperplasie, 
wie sie Barbacci.(1. c.) als Ausgang akuter, gelber Leberatrophie beschreibt, deren Knoten aus unregelmäBigen Lobulationen mit Verlust des acinösen Aufbaus bestehen, ist hier nicht vorhanden. Auch multiple Adenome sind auszuschlieBen (Literatur und Differentialdiagnose vgl, Barbacci, l. c.).

Trotz einiger Unterschiede bietet unser Fall auffallende Ähnlichkeiten mit Veränderungen, wie sie Marchand und Ströbe (ausfiihrliches Referat bei Barbacci, l. c) nach ausgedehnter parenchymatöser Degeneration beschreiben.

Somit ist es nach dem anatomischen Bilde am wahrscheinlichsten, daß wir die Folgen einer ausgebreiteten toxischen ${ }^{1)}$ (Pilzvergiftung?) oder infektiösen (Petrischalen, die bei der Sektion aus der Milz gegossen wurden, blieben steril) Degeneration des Leberparenchyms vor uns haben, wenn auch nicht völlig sicher auszuschließen ist, daß auch eine abgelaufene akute, gelbe Leberatrophie oder selbst die in ihren Erscheinungen so äußerst wandelbare Lues einmal ähnliche Bilder hervorzubringen vermögen.

Über die Ätiologie der Erkrankung hat die anatomische Untersuchung keinen sicheren Aufschluß geben können.

Wir haben die "starke Degeneration des Leberparenchyms" als die anatomische Grundlage unserer weiteren Beobachtungen anzusehen.

Wie änßerte sich die Degeneration des Leberparenchyms im Leben?

Welches Krankheitsbild verursachte sie? Welche Veränderungen des Stoffwechsels waren an den Ausscheidungsprodukten zu beobachten?

Welche Produkte pathologischen Stoffwechsels fanden sich in den Geweben post mortem?

Wie verhieit sich die Leber in bezug auf die Autolyse?

Klinische Beobachtung (Ibrahim).

Als Grundlage des klinischen Bildes geben wir im folgenden einen kurzen Auszug aus der Krankengeschichte.

Kind Heinrich Iser, 4 Jahre alt. Eintritt in die Kinderklinik am 13. November 1901 .

A $\mathrm{n}$ a m nes e: Gesunde Eltern. Keine luetische Infektion. Eine gesunde Schwester. Bisherige Erkrankungen: Mit 2 Jahren Lungenentzündung. Vor 5 Wochen Masern.

1) Bemerkt mag werden, daß im hiesigen pathol. Institut in den letzten Jahren ein Fall von chronischer Phosphorvergiftung bei einer Geisteskranken beobachtet worden ist, deren Leber in manchen Punkten eine interessante Ähnlichkeit hat. In unserem Falle lag kein Phosphorverdacht vor. 
Beginn der Erkrankung vor 14 Tagen: Obstipation. Appetitlosigkeit. Gelbsucht. Lehmige Stühle. Dunkler Urin. In den ersten Tagen der Erkrankung blutiges Erbrechen, jetzt öfters Nasenbluten, nie Blut im Stuhl. Seit 2 Tagen Verschlimmerung: Unrube, viel Schreien, Benommenheit. Ätiologisch nichts feststellbar, kein Trauma, Vergiftungsmöglichkeit nur für Pilzvergiftung vorhanden.

Status praesens: Leidlicher Ernährungszustand. Sehr schwerer Krankbeitseindruck. Starker Ikterus. Hautblutungen, keine Ödeme. Mitunter Nasenbluten. Leichte Bronchitis. Herz ohne Befund.

Puls: verlangsamt, hart, $60-80$.

A bdomen: kein Meteorismus, kein Ascites, kein Tumor füblbar.

Leber: druckempfindlich, rechter Leberlappen hart, glatt, vergrößert, in der Mammillarlinie 1 Querfinger mit dem Rippenbogen palpabel. Dämpfungshöhe $7 \mathrm{~cm}$. - Linker Leberlappen, hart, glatt, eher verkleinert.

Milz: vergrößert $11,5: 6,5$, weich, palpabel.

Stuhl: gallenarm, keine Parasiteneier.

Urin: sauer, bierbraun. Gmelin pos., Gallensäuren stark pos., Eiweiß und Zucker negativ. Azeton und Azetessigsäure negativ. Kein Leuzin oder Tyrosin.

Mikroskopisch: Ikterisch verfärbte Epithelien und Leukozyten,

Temperatur: subnormal.

Nervensystem: Sopor. Mitunter Schreianfälle und große motorische Unruhe. Normaler Atemtypus. Kein meningealen Erscheinungen. Normaler Augenhintergrund. Alle Reflexe herabgesetzt.

15. November: Stühle immer ganz dünn. Urin: Spur Alb., Azet. essigsäure pos. $400 \mathrm{~g}$ phys. Kochsalzlösung subkutan.

16. November: Leber wie bisher, Milz 10:7. Verdacht anf beginnenden Aszites. Meteorismus. Auf Santonin kein Askarisabgang. Urin: Alb. stark + , Zucker + (Gärangsprobe). Azeton nnd Azetessigsäure +. Mikroskopisch: $2 \mathrm{granulierte} \mathrm{Z} \mathrm{Ylinder.} \mathrm{Blut:} \mathrm{Gerinnungsfähigkeit} \mathrm{un-}$ verändert, rasche Stechapfelbildung. Geringe Leukozytose. Bouillonkulturen bleiben steril. Verhältnis des $\mathrm{NH}_{3}$ Stickstoffs zum Gesamt-N im frisch dureh Katheter entnommenen Urin $=10,9: 100$.

17. November: Ikterus nimmt ab, Sopor zeitweise geringer - 0 deme der Handrücken und Bauchdecken. Neue Hautblutungen. Meteorismus und deutlicher Aszites. Urin nach Gaben von $6 \mathrm{~g} \mathrm{Natr.} \mathrm{carbon.}$ und $6 \mathrm{~g}$ Natr. bicarbon, beständig sauer. Eiweiß nur in Spuren, kein Azeton und Azetessigsäure, Zucker. Reichlich Leuzin.

18. November: 2,5 Natr. bicarb. Urin bleibt sauer. Blut: Gerinnbarkeit gut. Kulturen bleiben steril. Leazin und Tyrosin. Kein Gallenfarbstoff.

19. November: Ödeme gewachsen. Punktion des Aszites (1100 g). Leber wird nicht palpapel. Dämpfungshöhe $7 \mathrm{~cm}$. - Milz $9: 6.6 \mathrm{~g}$ Natr. carbon. Urin bleibt sauer, keine Spur Alb., Azeton nnd Azetessigsäure, deutlich pos. Fieber: 38,5.

20. November: Bronchitis. Beiderseitige Otititis eatarrhalis. Ikterus verschwunden. Erbrechen und Zähneknirschen. Urin: sauer, Azetessigsäure pos., kein Alb., kein Zucker.

21. November: Zerebrale bezw. meningeale Erscheinungen: Papillen- 
differenz, Nackenstarre, Kan- und Saugbewegung. Automatische Armund Beinbewegung. Zungendeviation. Große Blutaustritte unter die Haut nach Einwirkung leichter Traumen. Regelmäßige tiefe Atmung.

22. November: Zunahme der Zerebralerscheinungen, kein Angenspiegelbefund. Tiefe Atemzüge in regelmäßiger Abwechslung mit mehreren oberfächlichen. Bronchopneumonische Herde. Leber scheint im Verlauf der letzten Tage kleiner geworden zu sein. Dämpfungshöhe gestern $6 \mathrm{~cm}$, heute $4,5 \mathrm{~cm}$. Urin enthält seit gestern wieder Gallenfarbstoff, Azeton und Azetessigsäure pos. Kein Leuzin nachweisbar.

Exitus um 10 h. 40 m. vormittags.

Fassen wir die gesamten Erscheinungen kurz zusammen, so ergibt sich folgendes Krankheitsbild:

Ein vierjähriger, aus gesunder Familie stammender Knabe erkrankte ohne Fieber an einem Ikterus, der zunächst nur durch blutiges Erbrechen und Neigung zu Nasenbluten sich von einem einfachen katarrhalisehen Ikterus untersehied. Dazu gesellte sich am 10. Krankheitstage Benommenbeit, motorische Unrube; der gesamte Krankheitseindruck wurde ein ernster. Am 12. Tage, bei der Aufnahme ins Krankenhaus, wurden multiple Hautblutungen konstatiert, der Sopor hatte zugenommen, ohne daß sonstige zerebrale Erscheinungen bestanden. Es fand sich eine leicht vergrößerte, harte, druckempfindliche Leber, leichte Milzschwellung, kein Aszites.

Der Urin, der täglich auf alle normalen Bestandteile und pathologischen Beimengungen untersucht wurde, enthielt außer Gallenfarbstoff und Gallensäuren nichts Abnormes.

Während der folgenden Tage zeigte die Leber erst keinerlei Änderung, später schien sie sich etwas zu verkleinern, besonders der linke Lappen, die Milz zeigte keine wesentliche Ändernng. Am 16. Krankheitstag stellte sich Aszites ein, zu dem sich am folgenden Tage Ödeme der Handrücken und Bauchdecken, später auch der Beine und des Skrotum gesellten. Lidödeme wurden nicht beobachtet. Der Aszites nahm rasch zu, es wurden $1100 \mathrm{~g}$ Aszitesflüssigkeit durch Punktion abgelassen, die sich rasch wieder ersetzten.

Der Ikterus nahm zunächst fast bis zu völligem Versohwinden $a b$, wurde erst in den letzten Lebenstagen wieder deutlicher; ähnlich verbielt es sich mit dem Gallenfarbstoff im Urin. Der Puls, bei der Aufnahme verlangsamt und bart, wurde später frequent und in der Spannung wechselnd.

Die Temperatur, bei der Aufnahme subnormal, stieg 4 Tage vor dem Tode an und blieb bis zum Tode subfebril.

Nephritische Erscbeinungen, die beim Eintritt fehlten, machten 
sich schon am nächsten Tage bemerkbar, am übernächsten wurden neben reichlichem Eiweißgehalt im Urin 2 granulierte Zylinder gefunden; in der Folge versehwand das Eiweiß wieder aus dem Urin und trat erst am Todestag wieder in Spuren auf. Zylinder waren nicht mehr vorzufinden. - Die hydropischen Erseheinungen sind nach der Reihenfolge ihres Auftretens und der Schnelligkeit ihres Entstehens bei völligem Mangel nachweisbarer Herzstörungen als nephritische Ödeme zu deuten. Ob die nervösen Reiz- und Lähmangserscheinungen der letzten Lebenstage auf die ödematöse Beschaffenheit der Hirnhäute zu beziehen oder als Symptome endogener Intoxikation zu deuten sind, ist nicht zu entscheiden.

Vom 15. Krankheitstage an wurden Azeton und Azetessigsäure im Urin nachgewiesen. Bis zum Tode blieben sie mit wechselnder Deutlichkeit nachweisbar; nur an einigen Tagen fiel die Reaktion negativ aus. Die saure Reaktion des Urins wurde auch durch Zufuhr von Alkalien in größeren Mengen nicht beeinflußt. Leuzin wurde am 17. und den folgenden Krankheitstagen, Tyrosin in größerer Menge am 18. Tage aus dem Urin dargestellt.

Zueker wurde durch Gärungsprobe im Urin nur einmal nachgewiesen, während Fehlingsche Lösung auch später öfter reduziert wurde. Das Blut des Patienten zeigte bei zwei Untersuchungen normale Gerinnungsfähigkeit.

Die gesamte Krankheitsdauer belief sich auf 23 Tage.

Die Ätiologie des Krankheitsfalles blieb ungeklärt. Die zweimal vorgenommene bakterielle Untersuchung des Blutes ergab negative Resultate.

Alle Vergiftungsmöglichkeiten wurden ron uns in Betracht gezogen. Für Phosphor ergab sich gar kein Anhaltspunkt. Der grüne Wandanstrich im elterlichen Hanse enthielt nach einer Untersuchung: des chemischen Laboratoriums der Universität kein Kupfer oder Arsen.

Die einzige gegebene Möglichkeit war die einer Pilzvergiftung, da nahe dem Hause viele Pilze wuchsen und die Kinder dort oft unbewacht spielten. Ein paar probeweise mitgebrachte Pilze erwiesen sich als ungiftige Arten (Geh.-Rat Pfister). Auch ist die Vergiftung mit Ammanita phalloides, die wohl allein in Betracht kommt, nicht wahrscheinlich.

Es soheint aus dem Sahlischen ${ }^{1)}$ Berichte über diese Vergiftung

1) Beitrăge zur Kenntnis der Schwammvergiftungen. Mitteilungen der naturforschenden Gesellschaft in Bern. 1. Heft. 1885. S. 1-50. 
hervorzagehen, dab eine "typische Fettleber", "wie wir sie z. B. bei akuter Phosphorvergiftung vorfinden", der charakteristische Leberbefund ist. Außerdem blieb das Blut in der Leiche flüssig, und die Totenstarre blieb ans. Auch diese Symptome wurden von uns nicht beobachtet.

Ferner fehlte bei Sahli der Ikterus und das Eiweiß des Harns, Blutungen wurden nicht beobachtet. Auch die klinischen Symptome sind völlig anders wie das von uns beobachtete Krankheitsbild.

\section{Stoffwechselversuch (Soetbeer).}

Man hat schon früher sein Augenmerk auf den ätiologischen $\mathrm{Zu-}$ sammenhang von schweren Lebererkrankungen und Azidose gerichtet. Noorden hat die Belege dafür bis zum Jahre 1893 in seiner Pathologie des Stoffwechsels zusammengestellt.

Die Beobachtungen der Ammoniakmengen bei akuter gelber Atrophie sind nicht sehr zahlreich und keineswegs übereinstimmend.

Besonders die älteren Autoren fanden bei akuter gelber Atrophie kleine $\mathrm{NH}_{3}$-Werte. Dann finden wir im Jahre 1889 Rosenheim 1), er untersucht bei 4 jährigem Kinde in einem Bruchteil des Tagesharns und findet 4,7 Proz. vom Gesamt-N als $\mathrm{NH}_{3}-\mathrm{N}$. Im Jahre 1891 findet Noorde ${ }^{2}$ ) in der 24 stündigen Harnmenge $10,14 \mathrm{~g}$ Stickstoff und 1,84 $\mathrm{g}$ $\mathrm{NH}_{3}$-Stickstoff, das sind 18 Proz. vom Gesamt-N.

$M u ̈ n z e r^{3}$ ) beobachtete bei akuter, gelber Leberatrophie einmal nur leicht vermehrtes $\mathrm{NH}_{3}$, aber kurz vor dem Tode bei zwei anderen Fällen derselben Krankheit waren 32,6 Proz. und 70 Proz. von Gesamt-N als $\mathrm{NH}_{3}$ vorhanden. Nicht in der Tagesmenge sondern in Einzelportionen.

Fränkel ${ }^{4}$ ) fand $\mathrm{NH}_{3}$ bis anf das Fünffache des Normalen gestiegen.

Seit Noordens Zusammenfassung 1893 ist anßer der oben zitierten $M$ ünz er schen Arbeit noch eine Untersuchung von $S$ e $n$ ator ${ }^{5}$ ) mit 3 Teilen Vermehrung des $\mathrm{NH}_{3}$ über die Norm erschienen. P. F. Richter ${ }^{6}$ ) fand in einem Fall akuter Atrophie 7,1-16 Proz. $\mathrm{NH}_{3}$, im anderen 7,3-8,7 Proz. $\mathrm{NH}_{3}$ (bezogen auf den Gesamt-N.)

Seitdem sind noch einige Publikationen über akute, gelbe Leberatrophie, auf die wir unten zurückkommen, erschienev. $\mathrm{NH}_{3}$ ist nicht bestimmt.

Unser Kranke schied am 16. November, dem 17. Tag der Erkrankung, in frischer Probe von Katheterurin 10,9 Proz. des Gesamt- $\mathrm{N}$ als $\mathrm{NH}_{3}$ aus (die Probe betrug $30 \mathrm{ccm}$ Harn).

Am folgenden Tage gelang es, den Gesamt-Nachtharn von

1) Rosenheim, Zeitschr. f. klin. Med, 1889. Bd. XV. S. 441.

2) Noorden, Pathol. des Stoffwechsels. S. 292. Badt Diss. Berlin 1891.

3) Münzer, dieses Archiv. 33. S. 164-167.

4) Fränkel, Fall von akuter Leberatrophie. Berliner klin. Wochenschr. 1892. S. 1255.

5) Senator, Charité-Annalen. 23, S. $330-342$.

6) P. F, Richter, Berliner klin. Wochenschr. 1896. S. 141. 
12 Stunden zu sammeln; er enthielt $0,961 \mathrm{~g}$ Stickstoff, davon $0,112 \mathrm{~g}$ Stickstoff als Ammoniak, gleich 11,6 Proz.

Am nächsten, dem 19. Krankheitstag, wurde die Gesamtmenge Stickstoff in 24 Stunden bestimmt. Sie betrug $1,24 \mathrm{~g}$ bei $170 \mathrm{ccm}$ Gesamtharn, davon waren $0,228 \mathrm{~g}$ als $\mathrm{NH}_{3}$ enthalten, gleich 18,5 Proz. vom Gesamt-N.

Am 19. November betrug die Harnmenge $186 \mathrm{ccm}$ in 24 Stunden, sie enthielt $0,938 \mathrm{~g}$ Stickstoff und $0,158 \mathrm{~g}$ Stickstoff als Ammoniak, das sind 16,79 Proz. Ammoniak.

Am 20. November enthielt die nur teilweise aufgefangene Menge Harn $1,047 \mathrm{~g}$ Stickstoff und $0,135 \mathrm{~g}$. Stickstoff als Ammoniak, das sind 13,22 Proz. vom Gesamt-N.

Am 22. November, einen 'Tag vor dem Tode, gelang es noch einmal, die Gesamtmenge Harn, $200 \mathrm{cem}$, aufzufangen. Sie enthielt 2,726 g Stickstoff und $0,347 \mathrm{~g}$ Stickstoff als Ammoniak, das sind 12,73 Proz.

Fassen wir die Resultate in einer Tabelle zusammen, so ergibt sich für die drei vollständigen Tage:

\begin{tabular}{|c|c|c|c|c|}
\hline Zeit & Ges.-N. & $\mathrm{NH}_{3}-\mathrm{N}$. & $\begin{array}{l}\mathrm{NH}_{3}-\mathrm{N} \text { in Proz. } \\
\text { vom Gres. }-\mathrm{N}\end{array}$ & $\begin{array}{l}\text { Eingefuhrte Alkali- } \\
\text { menge }\end{array}$ \\
\hline 18. November & 1,24 & 0,228 & 18,5 & $\begin{array}{l}2,5 \text { Natr. carbon. } \\
3,0 \text { Natr. bicarb. }\end{array}$ \\
\hline 19. November & 0,938 & 0,153 & 16,8 & $\begin{array}{l}\text { 3,0 Natr. carbon. } \\
3,0 \text { Natr. bicarb. }\end{array}$ \\
\hline 22. November & 2,726 & 0,347 & 12,7 & 2,0 Natr. carbon. \\
\hline
\end{tabular}

Wir ersehen aus dieser Tabelle, wie die Ammoniakwerte eine abfallende Tendenz zeigen; $a b$ das der Medikation zuzusehreiben ist, ist wahrscheinlich, aber nicht sicher, es bätten wohl bedeutend größere Alkalidosen ruhig gegeben werden können, als es in Wirklichkeit geschehen ist. So blieb immerhin der prozentuale Gehalt an $\mathrm{NH}_{3}$ noch sebr hoch, wenn er auch nicht die hohen Zahlen der oben zitierten untersuchten Einzelportionen der akuten gelben Leberatrophie erreichte.

Man hat nun früber verschiedentlich versucht, auf experimentellem Wege die hohen Ammoniakzahlen zu beeinflussen, um so über ihren Ursprung ins Klare zu kommen.

Münzer (1. c.) beobachtete, daß bei Eingabe von Soda das Ammoniak sich von 16,5 auf 6 Proz. herunterdrücken ließ. W eintraud 1 ) beobachtete bei Fällen von Leberzirrhose mit Cholämie 7,5 Proz, 8,4 Proz. Ammoniak,

1) Weintrand, dieses Archiv, 31. S. 30. 
bei gutartiger Form derselben Krankbeit jedoch ganze normale Ammoniakzahlen. Sowohl Münzer wie Weintraud versuchten es, die $\mathrm{NH}_{3}$-Ausscheidung durch Eingabe von zitronensaurem Ammoniak zu beeinflussen.

$\mathrm{M} u ̈ n z e r$ fand keine Zunabme des $\mathrm{NH}_{3}$ wohl aber Zunabme des Gesamt-N. Dagegen fand Weintraud in den zitierten Untersuchungen, daß 4,886 g Ammoniak die Ammoniakmenge von 8,6-7,9 anf 15,6 Proz. steigen ließ. Die experimentellen Untersuchungen sind noch sehr spärlich, und ein sicherer Schluß läßt sich noch nicht schließen. Man hat andere indirekte Wege eingeschlagen, um die Säuerung nachzuweisen.

$\mathrm{K}$ ra us 1 ) untersuchte im Jahre 1889 das Blut auf seinen $\mathrm{CO}_{2}$-Gehalt bei Phosphorvergiftung und fand einen Gehalt des Blutes von 17, 18 Volumenprozenten $\mathrm{CO}_{2}$ gegen $31,17-35,96$ Proz. in der Norm. Sehon im Jahre 1881 hatte Hans Meyer ${ }^{2}$ ) an Kaninchen nachgewiesen, daß bei Phosphorvergiftung das Blut 50-80 Proz. seiner Alkaleszens einbüßt, die Methode war dieselbe wie die von $\mathrm{Kr}$ a us angewandte.

Richters(l. c.) Titration des Blotes, die bei akuter Leberatrophie viel Säure konstatierte, scheint mir nicht beweisend.

Schließlich ging man direkt auf das Ziel los und suchte nach organischen Säuren im Harn. Es gelang mehreren Autoren $\left.{ }^{3}\right)$ Fleischmilchsäure darzustellen, ferner wiesen andere 4) đie Oxymandelsäure nach.

Seit der Noordensehen Zusammenfassung ist von diesen Säuren niehts mehr dargestellt mit Ausnahme eines Falles von Leberzirrhose von Weintraud, die Milchsäure ausschied, die als $0,6 \mathrm{~g}$ milchsaures Zink gefunden wurden.

Außerdem finden wir bei $\mathrm{S}$ e $\mathrm{n}$ ator in der oben zitierten Untersuchung die Angabe, daß Azeton und Azetessigsäure im Harn bei akuter, gelber I Leberatrophie von ihm gefunden sein.

Wir haben nirgends sonst eine ähnliche Angabe gefunden, können aber die Senatorsehe Beobachtung durch eine zweite bei unserem Falle von ,akuter Degeneration des Leberparenchyms" bestätigen. Wir fanden starke Gerhardtsche Reaktion und Azeton im Harn, und um das hier gleich zu erwähnen, auch Zucker. (Fehling, Gärung) ein Vorkommnis bei akuter Lebererkrankung, fur das wir in der Literatur $\left.{ }^{\circ}\right) 2$ Parallelfälle finden konnten.

Und die Wahrscheinlichkeit der Säuerung der Gewebe zu stützen

1) Kraus, Zeitschr. f. Heilkunde. Bd. X, 1-57. 1859.

2) Hans Meyer, Phosphor. Dieses Archiv. Bd. XIV. 1881. S. 335.

3) von Noorden, 1. c. S. 294.

4) Derselbe, l. c. S. 298.

5) Naunyn, Diabetes melitus. Wien 1900. S. 39: 1 Fall von akuter gelber Leberatrophie, 1 Fall von Phosphorvergiftung mit Glykosurie. S. 48: Leberdiabetes mit Oxybuttersăure, 3 Fälle mit Eisenchloridreaktion. - Jaksch, Vergiftungen. Wien 1897. S. 148 sah nie Zucker bei Phosphorvergiftung. Von 40 Fallen 8 positive oder "schwachpositive" Azetonreaktion. 
Ũber einen Fall von akuter Degeneration des Leberparenchyms.

haben wir neben den Ammoniakbestimmungen die Gesamtmenge der vorhandenen Säuren und Basen analysiert.

Die Resultate geben wir in Tabelle II:

\begin{tabular}{l|l|l|l|l|l|l|l|l|l}
\hline \hline Datum & $\mathrm{NH}_{3}$ & $\mathrm{Na}$ & $\mathrm{K}$ & $\mathrm{CaO}$ & $\mathrm{MgO}$ & $\mathrm{U}$ & $\mathrm{P}_{2} \mathrm{O}_{5}$ & $\mathrm{Cl}$ & $\mathrm{SO}_{3}$ \\
\hline \hline 19. November & 0,158 & 0,107 & 0,027 & 0,019 & 0,006 & 0,096 & 0,200 & 0,137 & 0,1138 \\
22. November & 0,347 & 0,447 & 0,502 & 0,089 & 0,012 & 0,025 & 1,038 & 0,200 & 0,2102 \\
\\
Aquivalen te Natrium. \\
19. November & 0,259 & 0,107 & 0,016 & 0,016 & 0,007 & 0,013 & 0,097 & 0,089 & 0,068 \\
22. November & 0,57 & 0,447 & 0,296 & 0,073 & 0,014 & 0,034 & 0,505 & 0,163 & 0,121
\end{tabular}

Die Summe der Basenäquivalente am 19. November betrug 0,404, die der Säuren 0,268. Es müssen also, da der Harn sauer (Lakmus) war, noch mindestens $0,136 \mathrm{~g}$ Äquivalente Na als Säuren im Harn enthalten sein, es $\mathrm{k}$ ö $n \mathrm{n}$ e n jedoch bedeutend melir vorhanden sein. Ebenso am 22. November. Die Summe der Basenäquivalente betrug 1,400, die der Säuren 0,823. Die Differenz beträgt also $0,577 \mathrm{~g}$, die als Säuren noch im Harn gewesen sein müssen.

Wir konstatieren diesen Basenüberschuß im Harn in Gemeinschaft mit den hohen Ammoniakwerten und schließen aus dem Zusammentreffen beider Faktoren, daß es sich um eine richtige Säuerung des Organismus handelt.

Quantitative Schlüsse können wir aus unseren Zahlen nicht ableiten. Wenn wir nun die Soda der Aufnahme und die Natriumzahlen der Ausgabe vergleichen, muß es uns klar werden, welch starke Ausscheidungsbehinderung bei der ganzen Erkrankung mitgespielt hat. Interessant ist es dabei, den Befund des Harns mit dem anatomischen Befund der Niere zu vergleichen.

Gi ierke schreibt: „Die Nieren sind sehr vergrößert, die Kapsel leicht abziehbar, Nierenfläche glatt und spiegelnd. Auf dem Durchschnitt sind Mark und Rinde regelmäBig abgegrenzt, die Papillenspitzen sind von gelber, fettiger Farbe, die Rinde etwas getrübt", und mikroskopisch findet er Trübung und Schwellung der Epithelien, "Wenn auch nur in bescheidenem Maße", "dazu finden sich die Epithelien sowohl beider Arten von gewundenen wie der geraden Harnkanälchen ziemlich allgemein mit deutlich granulärem Fett erfüllt. Stärkere desquamatative oder interstitielle Prozesse fehlen."

Wir wissen zu wenig von der anatomisehen Veränderung des Parenchyms der Nieren und ihrer pathologisehen Funktion, um ein Urteil über den Zusammenhang der hier gefundenen anatomischen 
Veränderungen und den gefundenen Funktionsstörungen za fällen. Der anatomische Befund mul als eine ganz leichte parenchymatöse Nephritis bezeichnet werden. Es soheint uns daher nach unseren heutigen Kenntnissen rom Zusammenhang von Funktion und Anatomie der Nieren kaum angängig, die schwere Ausscheidungsstörung: allein auf das ausseheidende Organ zu wälzen. Es ist möglich, dab die Stoffe nicht harnfähig die Nieren passiert haben, dal schwere Bindungsanomalien vorliegen. Für sehwere Störungen der Ausscheidungsfunktion im Harn sprechen schon die Harnbefunde am 16. November, wo bei reichlicher $\mathrm{H}_{2} \mathrm{SO}_{4}$ und $\mathrm{Cl}$ keine Phosphorsäure nachzuweisen war. Am 17. November, wo deutlich $\mathrm{H}_{2} \mathrm{SO}_{4}$ und $\mathrm{Cl}$ wiederum keine Phosphorsäure im ganzen Nachtharn erschien. Am 18. November war immer noch keine Phosphorsäure im 24 stündigen Harn vorhanden. Am 19. November erschien endlich $0,2 \mathrm{P}_{2} \mathrm{O}_{5}$ durch Wägungsanalyse bestimmt. Ebenso sind alle drei Mineralsäuren am 20. November $z u$ finden. Am 22. November steigt die Phosphorsäure auf $1 \mathrm{~g}$. Wir haben schon in einer früheren Arbeit ${ }^{1}$ ) darauf hingewiesen, daß bei den Nephritiden sicher auch Anomalien der Bindung in den Geweben bei der beobachteten Ausscheidungsanomalie mitsprechen.

Es wäre von großem Interesse gewesen, za konstatieren, welehe organische Säure im Harn entbalten war. Der positive Ausfall der Gerhardschen Reaktion, das Vorhandensein von Azeton sprach für die Anwesenheit von Azetessigsäure und Oxybuttersäure.

Es gelang mit den geringen Mengen, die uns nur zu Gebote

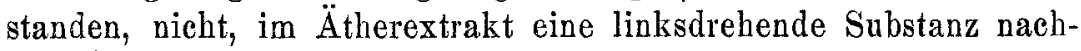
zuweisen.

Auch die Versuche, Fleischmilebsäure darzustellen, mißlangen.

Es ist nicht wunderbar, daß aus den geringen Überbleibseln des spärlichen und nicht sehr konzentrierten Harns nach Entnahme der vielen Analysenmengen für die Aschenanalyse nicht genügend Material für den direkten Nachweis und die Identifizierung organischer Säuren übrig blieb.

Noorden ist auf Grund eigener Beobachtung der Ansicht, daß starker toxiseher Eiweißzerfall bei akuter gelber Leberatrophie das gewöhnlichste sei. Es ist bei der meistens bestehenden Nephritis äußerst schwer über den N-Stoffwechsel ein Urteil zu fällen. Jedenfalls müssen wir wohl vorsichtiger behaupten, es findet keine $\mathrm{N}$-Ausscheidung oder eine starke $\mathrm{N}$-Ausscheidung an einzelnen

1) Soetbeer, Die Sekretionsarbeit der kranken Niere. Zeitschr. f. physiol. Chemie. 1902. 
Tagen (es sind überhaupt doch erst ein paar 24 stündige Versuche bei akuter, gelber Leberatrophie gemacht) statt. - Und zwar aus den verschiedensten Gründen.

Wir müssen bei allen diesen Betrachtungen uns vergegenwärtigen, daß die Nahrungsaufnahme und wohl auch besonders bei dem bestehenden Ikterus die Nahrungsausnutzung eine sehr schlechte war. Immerbin sind die genan in unserem Falle aufgeführten Nahrungsmengen so groß, daß die angeführten Stickstoff und Aschenmengen weit größer hätten sein müssen.

Wir stellen hier noch einmal die annähernd berechneten Stickstoff- und Kalorienwerte zusammen.

In 24 Stunden am 18. November nahm Patient auf einen Eßlöffel Gerstenmehl

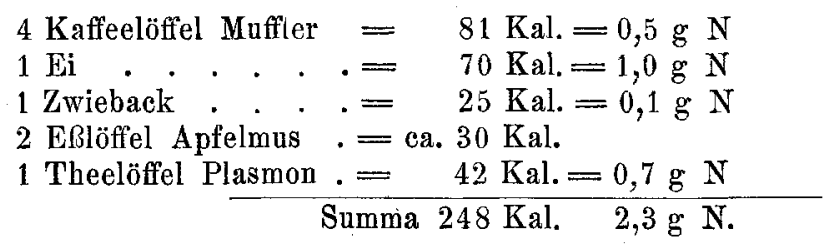

In 24 Stunden am 21. November. Per Klysma 1 Ei. Außerdem Schlundsonde:

2 Kaffeelöffel Muffler . $=40 \mathrm{Kal} .=0,25 \mathrm{~g} \mathrm{~N}$

$220 \mathrm{~g}$ Milch . . . . $=150 \mathrm{Kal} .=1 \quad \mathrm{~g} \mathrm{~N}$

4 Eier . . . . . . $=280$ Kal. $=4$ g N

$11 / 2$ Kaffeelöffel Plasmon $=63 \mathrm{Kal} .=1,5 \mathrm{~g} \mathrm{~N}$

Summa 533 Kal. $\quad 6,75 \mathrm{~g} \mathrm{~N}$

Am 18. November bei Mittelgewicht von $15 \mathrm{~kg}$ :

pro Kilo 16 Kal. Bruttoaufnahme.

Am 21. November:

pro Kílo $35 \mathrm{Kal}$. Bruttoaufnahme.

Die Quantität des aufgenommenen Stickstoffes läßt sich wegen der Ungenauigkeit der Berechnung den fehlenden Kotanalysen nicht zur Stoffwechselbilanz mit dem Harnstickstoff zusammenstellen. Das wäre aber auch bei der bestehenden Ausscheidungsanomalie ganz zwecklos. Jedenfalls aber kann man aus dem vorliegenden Material folgern, daß eine stärkere Stickstoffmenge im Harn nicht zur Beobachtung kam, was wohl Sehuld der Ausscheidungsanomalie ist.

Schließlich bleibt es nur noch übrig, auszuführen, daß es uns, wie den meisten neueren Autoren ${ }^{1}$ ) bei akuter gelber Leberatrophie,

1) Albu, Deutsche med. Wochenschr. 1901. S. 217. Akute gelbe Leberatrophie mit Heilung. Leuzin und Tyrosin, das letztere verschwand schneller. 
die leider ihr chemisch analytisches Interesse fast ausschließlich auf diesen Nachweis konzentriert haben, gelungen ist, Leuzin und bald darauf auch Tyrosin nachzuweisen.

Zusammenfassend rekapitulieren wir, daß es in dem vorliegenden Fall akuter Degeneration des Leberparenchyms gelang, durch Aufstellung der gesamten anorganischen Säuren und Basen des Harns an zwei Tagen nachzuweisen, daß vom Organismus abnorme Säuren ausgeschieden werden. Gestützt wird diese Anschauung durch hohe Ammoniakwerte trotz täglicher reicher Gaben von Soda und Natr. bircarbon. Über die Natur der Säuren gelang es nicht, sicheres nachzuweisen, einen Fingerzeig gibt die positive Gerhardtsche Reaktion und der Azetonbefund im Harn.

Über den N-Umsatz läßt sich aus dem Harnbefund nichts reduzieren, die N-Ausscheidung ebenso wie die Mineralstoffausscheidung war stark behindert. Wir wissen nicht, ob die Behindernng in der Beschaffenheit der anderen Gewebe oder der Niere liegt, glauben aber, daß die anderen Gewebe an der Ausscheidungsbehinderung: zum mindesten stark beteiligt sind.

Es war nun bei der darniederliegenden Ausscheidung für uns sehr hoffnungsreich, an die Durchforschung der Gewebe post mortem zu gehen.

Unsere Erwartungen wurden enttäuscht. Um ganz frisches Material sicher zur Verarbeitung zu bekommen, wurde die Sektion statim post mortem gemacht und die frischen Organe den betreffenden Herren Mitarbeitern sof ort zur Verarbeitung übergeben und anch sofort verarbeitet.

Anschließend an die Beobachtungen Salkowskis ${ }^{1}$ ), Röh manns ${ }^{2}$ ), Miuras ${ }^{3}$ ) und anderer ${ }^{4}$ ) unternahm es Cohnheim, die Gewebe auf ihre Eiweilsstoffe zu untersuchen, nachdem wir vorher die Abwesenheit jeglicher Albumosen im Harn konstatieren konnten.

Reichliche Indikan. - Aufrecht, Zentralbl. f. inn. Med. 1896. Nr. 11. S. 273. Am 3. Tag Leuzin bei akuter gelber Leberatrophie - A. Ibrahim, Zur Kenntnis der akuten Leberatrophie. Münchener med. Wochenschr. 1900. Macleau, Brit. med. Journal. 1898. Mai 28. Leuzin und Tyrosin gefunden bei akuter gelber Leberatrophie. - Thurnwald, Wiener med. Wochenschr. 1901. Nr. 29. 4 Tage vor dem Exitus Leuzin und Tyrosin. Sektionsbefund.

1) Salkowski, Virchows Archiv. 88. S. 394. 1882.

2) Röhmann, Berliner klin. Wochenschr. 1888. S. 862.

3) Miura, Virchows Archiv. 101. S. 316. 1885.

4) Jaksch, Zeitschr. f. klin. Med. 6. - Maixner, Prager Vierteljahrsschrift. 1879. 143. S. 75 . 
Über die Eimeißstoffe des Aszites des Bluts und der Leber (Cohnbeim).

1. Untersuchung der Aszitesflüssigkeit. Sie war eine opaleszierende Flüssigkeit, in der sich beim Stehen ein kleines Gerinnsel abschied. Die Eiweilsstoffe werden durch Kochen mit Essigsäure unter Zusatz von Kochsalz entfernt. Das wasserklare Filtrat gab keine Biuretreaktion und keine Fällung mit Phosphorwolframsäure. Zwei Proben der Aszitesflüssigkeit wurden bei sehwach alkaliseher Reaktion und unter Zusatz von Salzsäure zwei Wochen in den Brutschrank gestellt; die Eiweilstoffe werden nicht verdant; nach der Koagulation waren Albumosen, Peptone oder weitere Spaltungsprodukte nicht nachweisbar, die Flüssigkeit enthielt also weder Pepsin noch Trypsin. $\mathrm{Zu}$ einer weiteren Probe setzte ich eine kleine Menge Magenpepton und stellte sie zwei Wochen in den Brutschrank. Die Biuretreaktion war nach dieser Zeit unverändert. Die Flüssigkeit enthielt also auch kein Erepsin.

2. Leber. Ein kleines Stïck Leber wurde mit Sand zu Brei verrieben und mit Kochsalzlösung extrahiert, dann die Eiweilsstoffe durch Koagulation entfernt. Das Filtrat war nieht völlig klar und zeigte eine ganz schwache Biuretreaktion, die aber wohl auf kleine, der Koagulation entzogene Eiweißreste zu beziehen war. Irgendwelche Mengen von Albumosen oder Peptonen waren jedenfalls nicht vorhanden.

3. Blut. Das flissige Blut wurde mit physiologiseher Kochsalzlösung versetzt und die Blutkörperehen dureh Zentrifugieren entfernt. Das Serum enthielt keine eiweibspaltenden Fermente und ergab nach Koagulation des Eiweib keine Biuretreaktion. Die Prüfungsmethode war dieselbe, wie bei der Aszitesflüssigkeit.

Die Untersuchung auf abnorme Eiweilospaltungsprodukte, die Biuretreaktion gaben, hatte also kein positives Resultat ergeben. Dr. Steudel unternahm es, die Gewebe nach Extraktivstoffen und Hexonbasen zu durchsuchen.

Die Extraktivstoffe und Hexonbasen in den Geweben (Steudel).

Zur Untersuchung, die sich besonders auf Extraktivstoffe und das Vorkommen von Hexonbasen erstrecken sollte, standen mir im ganzen zur Verfügung: 110.g Leber, $750 \mathrm{~g}$ Muskeln (Körper- und Herzmuskulatur), $75 \mathrm{~g}$ Milz, 21 Aszitesflisssigkeit und 11/2 1 Blut.

Von diesen wurden die Aszitesflüssigkeit und das Blut nach 
Verdiunnung mit destilliertem Wasser bei schwach essigsaurer Reaktion koaguliert, filtriert und das biuretfreie, farblose Filtrat passend cingeengt. Die Organe wurden mit der Hackmaschine zerkleinert, dreimal mit siedendem, schwach essigsaurem Wasser extrahiert und die klaren, hellgelben Filtrate ebenfalls eingeengt bis auf $100-150 \mathrm{ccm}$.

Nun wurden sämtliche Flüssigkeiten zunächst mit Bleiessig' unter Vermeidung eines Überschusses gefällt, vom spärlichen Niederschlage filtriert und aus dem Filtrate das Blei mit Sehwefelwasserstoff entfernt. Das Muskelextrakt wurde dann bis zur Sirupdieke eingeengt und erstarrte nach einiger Zeit zu einem festen Kuchen von Kreatin. Die übrigen Extrakte wurden mit Schwefelsäure angesäuert und mit Phosphorwolframsäure eine Fällung zu erhalten versucht, aber es ist mir in keinem Falle geglückt, einen nur irgendwie nennenswerten Niederschlag zu erhalten, meist blieb auf Zusatz der Phosphorwolframsänre die Flïssigkeit vollkommen klar, nur bei dem Leberextrakt trat eine schwache Opaleszenz ein. Es wurde also die Schwefelsäure und die Phosphorwolframsänre wieder aus den Extrakten mit Barytwasser, der überschüssige Baryt mit Schwefelsäure entfernt und zum Sirup eingeengt, aber es kristallisieren nur wenige spärliche anorganische Kristalle ans, selbst nach langem Stehen.

Da nun sämtliche Reste eine intensive Rotfärbung mit Eisenohlorid zeigten, habe ich versucht, etwa vorhandene $\beta$-Oxybuttersäure zu finden. Es wurden also die vereinigten Extrakte aus Blut und Aszites mit Schwefelsäure wieder angesänert und mit ̈̈ther sorgfältig extrahiert, dabei gingen aber nur Spuren von Substanz in den Äther binein, der Ätherextrakt war optisch völlig inaktiv, und der Rückstand zeigte noch immer die Eisenchloridreaktion, die dem $\ddot{A}$ therextrakt völlig fehlte.

Eine weitere Verarbeitung der Reste war vollkommen aussichtslos, dazu war ihre Menge eine gar zu geringe, die geringe Menge des Ausgangsmaterials ist auch der Grund, weshalb ich dem negativen Ausfall meiner Versuche keinen allugroßen Wert beilegen zu sollen glanbe; sichere und zuverlässige Resultate über das Vorhandensein und die Menge der Extraktivstoffe usw. werden sich nur dann gewinnen lassen können, wenn das Ausgrangsmaterial in solchen Massen zugänglich sein wird, daß sich die einzelnen Körper daraus in kristallisiertem Zustande isolieren lassen. Im vorliegenden Falle handelt es sich aber nur um die ,Verarbeitung weniger Kubikzentimeter, trotzdem fast sämtliches verfitgbares Material in Arbeit genommen war. Nun ist aber die Beschaffung großer Mengen menschlicher 
Organe mit denselben oder gleichmäßigen, pathologischen Veränderungen, besonders direkt post mortem, um sicher bei der nachfolgenden Untersuchung nur intravitale Veränderungen vor sich zu haben, mit großen Schwierigkeiten verknüpft und weitere Untersuchungen in größerem Umfange, auf Grund deren dann ein endgültiges Urteil abgegeben werden kann, mlissen solange verschoben werden, bis das nötige Material gesammelt sein wird.

Schließlich unternahm es Jacoby, die Leber auf ihr autolytisches Verhalten hin zu prïfen.

$$
\text { Die Autolyse der Leber (Jacoby). }
$$

Bei Untersuehung der Leber von Hunden, die mit Phosphor vergiftet waren, hatte sich ergeben (s. Zeitschr. f. physiolog. Chemie, Bd. 30, 1900), daß der Amid-Stickstoff und zwar insbesondere der direkt als Ammoniak mit Magnesia direkt austreibbare gegen die Norm stark vermehrt war. Namentlich wurde bei der Autolyse viel mehr derartiger $\mathrm{N}$ gebildet als in der Norm.

Die folgenden Tabellen geben die Daten solcher Versuche. Der direkt austreibbare Amid- $\mathrm{N}$ wird in Prozenten des Gesamt- $\mathrm{N}$ wiedergegeben, die Dauer der Autolyse beträgt stets 14 Tage.

A.

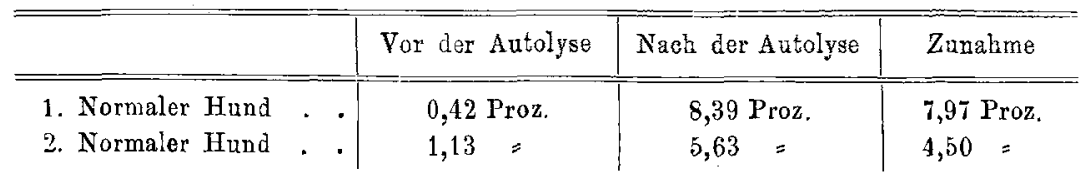

B.

1. Tod 36 Stunden nach Phosphorvergiftung keine typische Leber.

2. Typische Phosphorleber

3. Typisehe Phosphorleber

\begin{tabular}{l|l|l} 
& \\
0,56 Proz. & 13,06 Proz. & 12,50 Proz. \\
$1,7=$ & $29,9=$ & $28,2=$ \\
$9,53=$ & $38,33=$ & $28,8=$
\end{tabular}

Es wurde nur in ähnlicher Weise die Leber dieses Falles gepruft. Dabei ergaben sich mehrere Sehwierigkeiten, die der Untersuchung keinen entscheidenden Wert zukommen lassen. Einmal konnte nicht vergleichsweise normale Menschenleber untersucht werden, sodann mußte die Autolyse aus äußeren Gründen auf 3 Tage beschränkt werden.

Die Methode der Untersuchung war folgende:

Die Leber kam etwa zwei Stunden nach der Sektion, nachdem sie bis dahin auf Eis aufbewahrt war, zur Verarbeitung, es wurde bestimmt: 
1. In einer Portion der Gesamt-N.

2. Eine zweite Portion (40 g) wurde mit $\mathrm{N}$-freiem Zinksulfat unter Zusatz von $5 \mathrm{ccm} 10$ prozentiger Schwefelsäure bis zur Ganzsättigung ausgesalzen, das Filtrat auf $500 \mathrm{ccm}$ aufgefüllt und in abgemessenen Teilen der mit Magnesia austreibbare $\mathrm{N}$ bestimmt.

3. Eine dritte Portion von $34 \mathrm{~g}$ wird entsprechend wie 2. behandelt, nachdem sie mit $100 \mathrm{cem} 0,9$ prozentiger Kochsalzlösung und Toluol drei Tage bei $35^{\circ}$ gehalten war. Die Gesamt-N-Bestimmung: ergab $4,76 \mathrm{~g} \mathrm{~N}$ in $100 \mathrm{~g}$ Leber. Von diesem Gesamt- $\mathrm{N}$ war als direkt austreibbarer Amid-Stickstoff vorhanden:

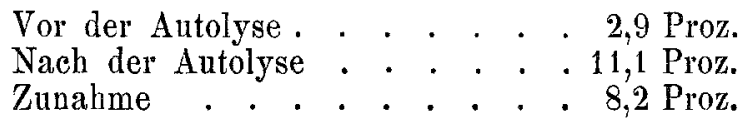

Der erhaltene Wert ist dem Anschein nach kein exzessiver. Ob analoge Steigerungen der Autolyse, wie sie bei der Phosphorvergiftung gefunden worden sind, sich bei Lebererkrankungen des Menschen finden, muß demnach unentsehieden bleiben, bis weitere Fälle untersucht sind und normale Leber verarbeitet worden ist.

Derartige Untersuchungen werden auch bei den schwierigen Verhältnissen der menschlichen Pathologie nicht aussichtslos bleiben, da das Beispiel der Phosphorvergiftung zeigt, daß die veränderten Organ funktion en unter Umständen noch nach dem Tode im isolierten Organ nachzuweisen sind.

Hingegen ist das Fehlen von Albumosen and Peptonen in den Organen schon darum nicht auffallend, weil diese Substanzen keine große Rolle beim Eiweißabban in den Zellen spielen; aber anch die kristallinischen Spaltungsprodukte, in welche das Zelleiweiß vorwiegend gespalten wird, brauchen nicht in den Organen angehäuft zu werden (s. d. Ausführungen in Zeitschr. f. phys. Chemie, Bd. 30, 1900).

Bemerkt sei hier noch, was in der eben zitierten Arbeit nicht besprochen worden ist, daß das vermehrte Auftreten von Ammoniak bei der Autolyse der Phosphorleber primär durch den Reiz einer abnormen Säurebildung verursacht sein kann.

\section{Sehlußwort.}

Wir haben es versucht, das Krankheitsbild der anatomisch fixierten, akuten Degeneration der Leber nach jeder Richtung hin getreu wiederzugeben. Vielleicht wird unser Vorgehen auch dadurch weiter Nutzen bringen, daß auch andere bekanntere und unbekannte Krankheiten in gleicher Weise an frischestem Material durchgearbeitet werden. 